\title{
Swelling of a granular hydrogel medium under confinement: individual and collective dynamic
}

\author{
Lucía Cristófaro ${ }^{1}$, Sebastián Falcioni ${ }^{1}$, Leonardo Binda ${ }^{1 *}$, Yanina Lucrecia Roht $^{1}$, Germán Drazer $^{2}$, and Irene Ippolito ${ }^{1}$ \\ ${ }^{1}$ Universidad de Buenos Aires, Facultad de Ingeniería, Grupo de Medios Porosos, C1063ACV, Buenos Aires, Argentina \\ ${ }^{2}$ Mechanical and Aerospace Engineering Department, Rutgers University, NJ 08854, New Jersey, EEUU
}

\begin{abstract}
The growth dynamics of polyacrylamide hydrogel beads due to liquid absorption under geometric confinement is studied. First, the behavior of individual hydrogel beads is investigated and, subsequently, the growth dynamics is analyzed in the presence of several interacting beads of the same material. In both, individual and collective studies, the magnitude of the geometric confinement is varied, in order to investigate the effect that the stress applied on the particles has on their hydration kinetics.
\end{abstract}

\section{Introduction}

In everyday life, as well as in different industries, granular materials such as powders, sands and coffee beans, are common. Sometimes these material surprise us with interesting mechanical behavior [1-3]. The behavior of soft, elastic, and frictionless particles, is distinct from that of hard grains and there are many open questions, including particle shape and deformation under stress. While some of the phenomena that distinguish soft particles qualitatively from hard grains can be easily explained, some features are less well understood. The role of softness, elasticity and friction on flowing soft granular particles through narrow holes has been studied in detail, including the characterization of the flow structure, and the measurement of pressure and velocity fields $[4,5]$. In particular, the refractive index matched scanning (RIMS) method has been successfully used to perform experiments in $3 \mathrm{D}$ dense granular samples $[6,7]$.

Hydrogel beads are interesting example of a soft granular material. Hydrogels are cross-linked polymeric networks that are insoluble but can absorb large amounts of water. Hydrogel beads are used in a wide variety of applications, ranging from humidity control to agriculture $[8,9]$. In many cases, the hydrogel beads are required to absorb liquid subjected to a force that opposes its growth [10].

Therefore, the behavior of hydrogel beads under confinement is of great importance to those applications. As a hydrogel bead absorbs water it swells significantly, and the mass transport of the absorbate is coupled with the mechanical deformation of the absorbent, which depends on the crosslinking of its network. The combination of properties of the absorbate and the elastic properties of the gel network makes it useful for different applications [11].
For small volume changes, the swelling dynamics of a hydrogel has been studied using both linear [8-14] and non-linear models [15-18]. However, the liquid transport mechanism within a hydrogel changes according to the internal porosity of the hydrogel studied. There are four typical mechanisms that control the growth of a hydrogel: diffusion, by chemical reaction, by swelling and by magnetic force [19].

Lin et al [20] studied hydrogels in confinement, compressing an individual hydrated hydrogel bead by an external uniaxial force. They model the deformation depending on the compressibility (incompressibility) of the hydrogel and assuming the beads remain axisymmetric for any deformation.

Here we consider the opposite situation in which the dry hydrogel beads are geometrically confined by two rigid plates before they are allowed to swell. As the hydrogel swells in contact with an aqueous solution its growth is eventually affected by the presence of the rigid plates. In this work we investigate the resulting growth dynamics for different apertures between the plates, $\mathrm{H}$. We consider both the growth of individual, isolated hydrogel beads as well as the collective growth of interacting beads. In the collective experiments growth is also constrained to central region between the plates, leading to bead-bead interactions and lateral compression forces that could also affect growth dynamics.

First, we present the size evolution of the hydrogel beads under confinement. We then analyze the asymptotic diameter and volume reached by the hydrogel beads as a function of the aperture H. Finally, we also analyze the growth kinetics. Throughout the work we compare the results obtained in individual bead experiments with those obtained in collective growth experiments.

\footnotetext{
* Corresponding author: lbinda@fi.uba.ar

A video is available at https://doi.org/10.48448/dfgf-p787
} 


\section{Experimental setup}

A transparent Hele-Shaw cell with a controlled aperture $\mathrm{H}$, between 2 and $15 \mathrm{~mm}$, was used to study the growth of (commercially available) spherical polyacrylamide hydrogel beads during the absorption process under geometric confinement. The mean diameter of the dry hydrogel beads is $2,8 \pm 0,2 \mathrm{~mm}$ and $15 \mathrm{~mm}$ when it is fully hydrated. Initially, the hydrogel beads were placed in the empty Hele-Shaw cell, which is then filled by water at the beginning of the experiments. A small amount of a soluble dye is added to the water (water blue, $1 \mathrm{~g} / \mathrm{l}$ ). The Hele-Shaw cell is illuminated from below and the dye allows us to visualize the growth of the hydrogels as a function of time using a CCD camera placed above the cell, as shown in figure 1. During experiments, images are acquired every two minutes. The hydrogels have the same refractive index as water but using the colored aqueous solution we were able to obtain good contrast between the edges of the hydrogels and the liquid.

As discussed in the introduction, we performed both individual and collective experiments. In the individual growth experiments, three hydrogel beads are placed in the cell, sufficiently separated from each other to avoid contact between them during the absorption process. We shall also assume that there are no hydrodynamic interactions between them. In collective growth, a constant number of 40 hydrogels was used. For these experiments, a $10 \mathrm{~cm}$ diameter silicone ring was added between the plates to contain the hydrogels in the plane of the cell. The hydrogels were initially distributed around the inner perimeter of the ring and the aqueous solution was injected from a center hole in the upper glass plate. The size of the ring was selected to ensure that the final distance between fully hydrated hydrogel beads is similar to their size, thus leading to significant bead-bead interactions without significantly hindering their total growth.

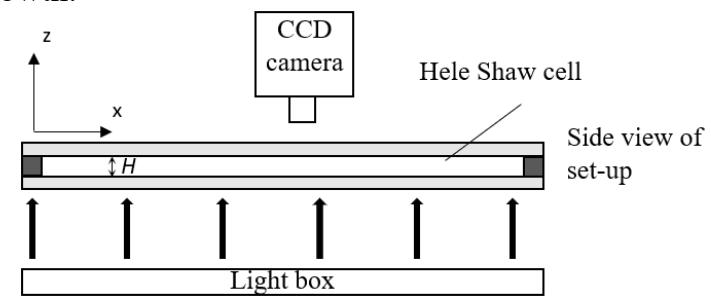

Fig. 1. Side view of the experimental setup. A horizontal HeleShaw cell with aperture H. The CCD camera acquires images of the intensity of light transmitted through the cell.

\section{Results and discussion}

\subsection{Hydrogel size as a function of time}

Using a standard image analysis method, we determine the size of the hydrogel beads as a function of time, for each value of the aperture $H$. In their final equilibrium state, the hydrogel beads make contact with the confining plates and we are able to identify and measure two characteristic diameters: the external diameter $\mathrm{D}_{\mathrm{Ext}}$, corresponding to the maximum diameter reached by the swollen hydrogel bead in the plane of the cell, and the contact diameter $\mathrm{D}_{\mathrm{C}}$, which corresponds to the diameter of the contact zone between the hydrogel bead and the walls of the cell. In figure 2, we show two images indicating these two diameters for a fully hydrated hydrogel bead inside a cell with aperture $\mathrm{H}=8 \mathrm{~mm}$. In the collective growth experiments, it is not possible to differentiate these diameters through image analysis. Therefore, we obtained a single diameter $\left(D_{M}\right)$ and we assume that it correspond to the mean value of the previous two, that is $D_{M}=\left(D_{E x t}+D_{C}\right) / 2$. This mean value is then used to compare the results in these collective growth experiments with those obtained in individual growth experiments.

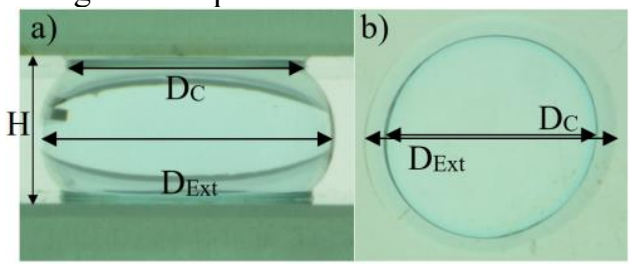

Fig. 2. Characteristics diameters (DExt y Dc) of a hydrated hydrogel under confinement, $\mathrm{H}=8 \mathrm{~mm}$. a) Side view. b) Top view.

In all the experiments we measured the diameter of individual beads in different directions in the plane of the cell, verifying that the growth of the hydrogel beads is isotropic in this plane. In figure 3, we present the values of the external diameter $D_{\text {Ext }}$ as a function of time obtained for (independent) individual growth experiments for the different values of the aperture $\mathrm{H}$ considered here. For comparison, we also present the growth dynamics of a hydrogel swelling in bulk water in the absence of any geometric confinement (free growth).

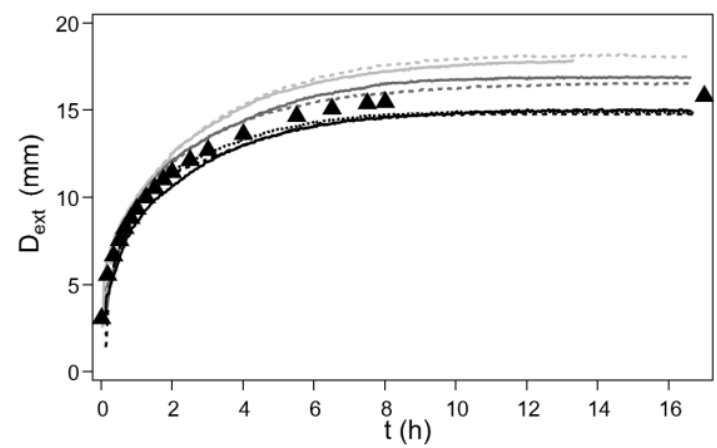

Fig. 3. $D_{\text {Ext }}$ of individual growth experiments as a function of time for all cell apertures $\mathrm{H} . \quad 2 \mathrm{~mm} ;-12 \mathrm{~mm}$; and $\quad . .15$ $\mathrm{mm}$. In $\boldsymbol{\Delta}$ the values obtained for the free growth of hydrogel.

First, we observe that the growth kinetics is similar in all classes, including that for free growth. However, we observe that the maximum external diameter reached by the hydrogel beads depends on the confinement.

In figure 4, we compare the equilibrium $\mathrm{D}_{\text {Ext }}$ results to the values obtained with the spherical approximation proposed by Lin et al (see equation (1) in [22]), using the contact diameter determined in our experiments. A good agreement is observed for relatively small values of the aperture. It is important to mention a significant difference with the study of Lin et al [20]: Lin et al. applied the confining stress after the hydrogel beads were 
fully hydrated. Here, in contrast, the spheres are always confined as they grow and reach an equilibrium state.

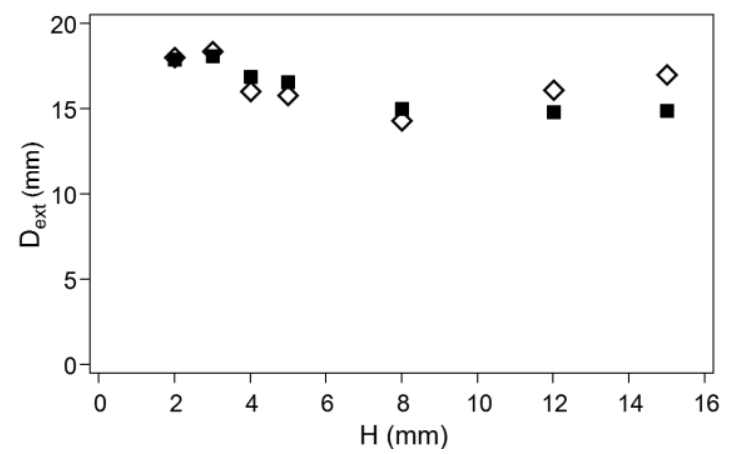

Fig. 4. DExt as a function of cell opening H. $\mathbf{c}$ correspond to individual growth experiments at end time, $\diamond$ calculated using the geometric spherical approach proposed by Lin et al [20].

Figure 5 compares the final mean diameter values $\mathrm{D}_{\mathrm{M}}$ obtained in individual and collective growth experiments. For individual growth experiments we plot the mean value over different realizations. In the case of collective growth experiments we plot the mean value calculated over all the hydrogel beads present in the cell. It is observed that in both cases, when the aperture $\mathrm{H}$ increases, the mean diameter $\mathrm{D}_{\mathrm{M}}$ decreases. In addition, it is clear that, for a given aperture, the mean diameter in the collective growth experiments is smaller than that obtained for individual hydrogel beads. This is due to the fact that growth is not only confined by the aperture of the cell $\mathrm{H}$ but also by the presence of neighboring hydrogel beads inside the ring.

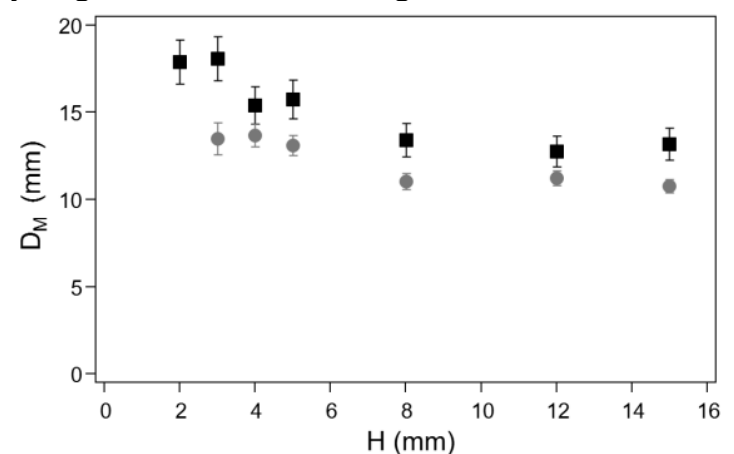

Fig. 5. $\mathrm{D}_{M}$ value of hydrogels reached at asymptotic time as a function of cell aperture H. $\bullet$ individual growth experiments, $\bullet$ collective growth experiments. The bars represent the standard deviation with respect to the mean value measured experimentally.

Let us note that figure 5 shows two distinct regions depending on the aperture $\mathrm{H}$ : for $\mathrm{H}$ smaller than $6 \mathrm{~mm}$, a clear decrease in $\mathrm{D}_{M}$ is observed, while for larger $\mathrm{H}, \mathrm{D}_{\mathrm{M}}$ remains nearly constant. That is, for relatively small values of the aperture, confinement has an appreciable influence on the equilibrium size of the hydrogel beads, but as the aperture of the cell increases, the equilibrium size of the hydrogel beads becomes independent of $\mathrm{H}$, as expected.

It should be noted that the equilibrium diameter without confinement (free growth) is higher than that obtained even under little confinement (largest values of $\mathrm{H}$ considered here).

\subsection{Final volume as a function of $H$ and deformation due to confinement}

To calculate the volume of the hydrogel beads, it was necessary to model their shape inside the cell. As we mentioned before, the approach for small apertures is based on the works of Lin et al [eq 5, 22]:

$$
V=2 \pi\left(\left(\frac{H^{2}}{4}+\frac{D_{C}^{2}}{4}\right)\left(\frac{H}{2}\right)-\frac{H^{3}}{24}\right) \text {. }
$$

And, for low confinement situations, that is larger $\mathrm{H}$ values, a good estimate is to approximate the hydrogel beads as ellipsoids:

$$
V=\frac{4}{3} \pi\left(\frac{H}{2}\right)\left(\frac{D_{E x t}}{2}\right)^{2} .
$$

We note that, to analyse collective growth experiments, the situation is different. In that case, we approximate the shape of hydrogel beads as cylinders with a diameter $D_{M}$ and the volume is therefore given by:

$$
V=H \pi\left(\frac{D_{E x t}+D_{C}}{4}\right)^{2} .
$$

In figure 6 , we present the equilibrium volume of the hydrogel beads in both individual and collective experiments as a function of the aperture of the cell.

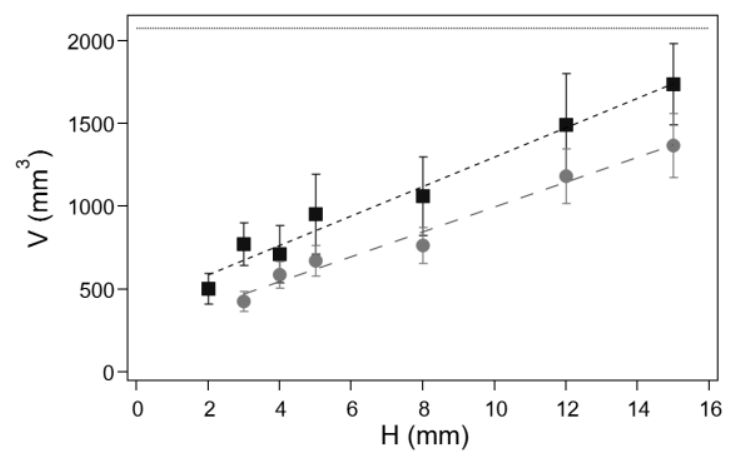

Fig. 6. Volume of hydrogels reached at end time as a function of $\mathrm{H}$ cell aperture. - individual growth experiments, collective growth experiments. In dotted line, the value for the hydrogel without confinement. The bars represent the measurement error that comes from the propagation of errors in the volume measurement of equations (1) to (3).

In both individual and collective growth experiments, the volume of the hydrogel increases as the $\mathrm{H}$ increases. The linear increase in volume with the aperture of the cell is consistent with equations (1)-(3), where the change in aperture dominates over the changes in diameter.

It can also be observed in figure 6 that, for a given aperture, the equilibrium volume reached by the hydrogel beads in the collective growth experiments is always lower than that obtained when they grow individually. This corresponds to what was previously observed in figure 5, where the mean diameter of hydrogels in collective experiments was below that of obtained in individual hydrogel bead experiments. As mentioned, in collective growth experiments, in addition to the confinement imposed by the cell, neighboring hydrogel beads generate confinement of elastic characteristics.

\subsection{Growth kinetics}

To study the kinematics of the hydrogels in both types of experiments, the average evolution of the external 
diameter $\mathrm{D}_{\text {Ext }}$ was considered. Specifically, we fitted the experimental data using the following exponential function of time:

$$
D(t)=D_{\text {Final }}-\left(D_{\text {Final }}-D_{\text {Initial }}\right) e^{-t / \tau},
$$

where $\tau$ corresponds to the characteristic tiem of the growth process. The initial value $D_{\text {Initial }}$ corresponds to $D_{\text {Ext }}$ at $t=0$, and the final value $D_{\text {Final }}$ was measured 11 hours after the beginning of the experiment, that is, when the hydrogels attain their maximum hydration. In figure 7 we present the results obtained for $\tau$ as a function of cell aperture $\mathrm{H}$.

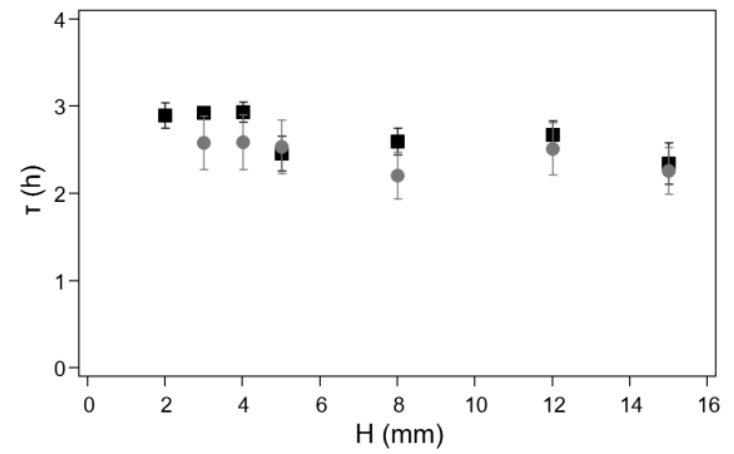

Fig. 7. Characteristic time $\tau$ as a function of the aperture of cell $\mathrm{H}$ obtained by equation (4) $\mathbf{n}$ individual growth experiments, $\bullet$ collective experiments growth. The bars represent the dispersion of $\tau$ for each experiment.

It can be observed that the characteristic time $\tau$ is nearly constant with $\mathrm{H}$, with the value for small apertures ( $\mathrm{H} \leq 5 \mathrm{~mm}$ ) slightly larger than that obtained for low confinement $(\mathrm{H} \geq 8 \mathrm{~mm})$. Furthermore, we observe that the growth kinetics has similar values for both types of experiments. Therefore, it is clear that the presence of interactions between hydrogel beads does not significantly influence the characteristic time of the absorption process, independent of the geometric confinement experience by the beads.

\section{Conclusion}

In the individual growth experiments, it was observed that the hydrogel beads grow symmetrically under onedimensional confinement. As the level of confinement decreases ( $\mathrm{H}$ greater), the external diameter reached by the particles decreases. In other words, for situations of low confinement, practically the same final diameter is reached.

In terms of growth kinetics, there are no significant variations depending on the aperture, obtaining characteristic growth times with a maximum variation of 35 minutes in an 8 hour process.

In the collective growth experiments, it is observed that the asymptotic equilibrium diameter reached by the hydrogel beads is smaller than those reached when they grow individually (without interaction between particles). This decrease in the bead diameter obtained in collective growth was found for all the apertures studied. This shows that collective effects are important, even in the presence of high geometric confinement. On the other hand, the influence of bead-bead interactions on the growth kinetics is very small. This is an important result when it comes to predicting behaviors in certain applications such as soils. Thus, in a confined situation, which is new, conclude that the hydrogel bead maintains its spherical structure despite growing confined. We notice this effectively up to $\mathrm{H}$ about $8 \mathrm{~mm}$. For larger $\mathrm{H}$ we need to use a growth model that takes into account an elliptical deformation one (because the confinement is smaller).

Finally, we mention that it would be interesting to study the internal pore structure (or porosity) of hydrogels, to understand the influence during the confined growth.

This work was supported by the University of Buenos Aires grant (UBACYT 20020170100225BA). L.B. is a fellow of a CONICET post-doctoral fellowship.

\section{References}

[1] H.M. Jaeger, S.R. Nagel, R.P. Behringer, Rev. Mod. Phys. 68, 1259 (1996)

[2] Y. Forterre, O. Pouliquen, Annu. Rev. Fluid Mech. 40, 1 (2008)

[3] D. Bi, J. Zhang, B. Chakraborty, R.P. Behringer, Nature 480, 355 (2011)

[4] X. Hong, M. Kohne, M. Morrell, H. Wang, E.R. Weeks, Phys. Rev. E 96, 062605 (2017)

[5] A. Ashour, T. Trittel, T. Börzsönyi, R. Stannarius, Phys. Rev. Fluids 2, 123302 (2017)

[6] J.A. Dijksman, N. Brodu, R.P. Behringer, Rev. Sci. Instrum. 88, 051807 (2017)

[7] J.A. Dijksman, H. Zheng, R.P. Behringer, AIP Conf. Proc. 1542, 457 (2013)

[8] M.J. Zohuriaan-Mehr, H. Omidian, S. Doroudiani, K. Kabiri, J. Mater. Sci. 45, 5711 (2010)

[9] Y.P. Lee, H.Y. Liu, P.C. Lin, Y.H. Lee, L.R. Yu, C.C. Hsieh, PJ. Shih, W.P. Shih, I.J. Wang, J, Y. Yen, C.A. Dai, Colloid Surface B 175, 26 (2019)

[10] A. Sannino, G. Mensitieri, L. Nicolais, J. Poly. Sc. 91, 3791 (2004)

[11] K.Y. Lee, J.A. Rowley, P. Eiselt, E.M. Moy, K.H. Bouhadir, D.J. Mooney, Macromolecules 33, 4291 (2000)

[12] C.Y. Hui, V. Muralidharan, J. Chem. Phys. 123, 154905 (2005).

[13] J. Wahrmund, J.W. Kim, L.Y. Chu, C. Wang, Y. Li, A. Fernandez-Nieves, D.A. Weitz, A. Krokhin, Z.B. Hu, Macromolecules 42, 9357 (2009)

[14] J. Yoon, S. Cai, Z. Suo, R.C. Hayward, Soft Matter 6, 6004 (2010)

[15] T. Bertrand, J. Peixinho, S. Mukhopadhyay, C.W. MacMinn, Phys. Rev. Appl. 6, 064010 (2016)

[16] F.P. Duda, A.C. Souza, E. Fried, J. Mech. Phys. Solids 58, 515 (2010)

[17] N. Bouklas, R. Huang, Soft Matter 8, 8194 (2012)

[18] M. Engelsberg, W. Barros Jr, Phys. Rev. E 88, 062602 (2013)

[19] R.S. Langer, N.A. Peppas, Biomaterials 2, 201 (1981)

[20] Y.L. Lin, D.M. Wang, W.M. Lu, S.Y. Lin, K.L. Tung, Chem. Eng. Sci. 63, 195 (2008) 